\title{
Global attractors for the coupled suspension bridge system with temperature
}

\section{Filippo Dell'Oro ${ }^{a}$ and Claudio Giorgi ${ }^{b * \dagger}$}

\section{Communicated by A. Miranville}

This paper deals with the long-term properties of the thermoelastic nonlinear string-beam system related to the wellknown Lazer-McKenna suspension bridge model

$$
\left\{\begin{array}{l}
u_{t t}+u_{x x x x}-\left(\beta+\left\|u_{x}\right\|_{L^{2}(0,1)}^{2}\right) u_{x x}+(u-v)^{+}+\theta_{x x}=f \\
v_{t t}-v_{x x}-(u-v)^{+}+\varphi_{x}=g \\
\theta_{t}-\theta_{x x}-u_{t x x}=h \\
\varphi_{t}-\varphi_{x x}+v_{t x}=0
\end{array}\right.
$$

In particular, no mechanical dissipation occurs in the equations, because the loss of energy is entirely due to thermal effects. The existence of regular global attractors for the associated solution semigroup is proved (without resorting to a bootstrap argument) for time-independent supplies $f, g, h$ and any $\beta \in \mathbb{R}$. Copyright $\odot 2015$ John Wiley \& Sons, Ltd.

Keywords: coupled bridge system; absorbing set; global attractor; regularity

\section{Introduction}

We are interested in scrutinizing the thermomechanical dynamics (in the transversal direction) of a string-beam model composed of an extensible thermoelastic beam with hinged ends coupled with a thermoelastic cable with fixed ends. The corresponding equations take the following form:

$$
\left\{\begin{array}{l}
u_{t t}+u_{x x x x}-\left(\beta+\int_{0}^{1}\left|u_{x}(x, \cdot)\right|^{2} \mathrm{~d} x\right) u_{x x}+(u-v)^{+}+\theta_{x x}=f, \\
v_{t t}-v_{x x}-(u-v)^{+}+\varphi_{x}=g, \\
\theta_{t}-\theta_{x x}-u_{t x x}=h \\
\varphi_{t}-\varphi_{x x}+v_{t x}=0
\end{array}\right.
$$

in the unknown variables

$$
u, v, \theta, \varphi:[0,1] \times[0, \infty) \rightarrow \mathbb{R}
$$

Here, $(u, \theta)$ and $(v, \varphi)$ are the (transversal) displacement-temperature pairs of the beam and the cable, respectively, and $\beta \in \mathbb{R}$ is a fixed constant accounting for the axial force acting on the reference configuration ( $\beta>0$ when the beam is stretched, and $\beta<0$ when it is compressed). In addition, $f, g, h \in L^{2}(0,1)$ are time-independent external sources: $f$ and $g$ stand for the lateral load distributions on the beam and the cable, respectively, while $h$ is the external heat supply to the beam. Finally,

$$
(u-v)^{+}=\max \{u-v, 0\}
$$

denotes the positive part of the function $u-v$. The system is complemented with the hinged boundary conditions for $u$

\footnotetext{
a Institute of Mathematics of the Academy of Sciences of the Czech Republic, Žitná 25, Praha 1115 67, Czech Republic

b Università degli Studi di Brescia, DICATAM - Sezione di Matematica, Via Valotti 9, Brescia 25133, Italy

* Correspondence to: Claudio Giorgi, DICATAM - Sezione di Matematica, Università degli Studi di Brescia, Via Valotti 9, Brescia 25133, Italy.

†E-mail: claudio.giorgi@unibs.it
} 


$$
u(0, t)=u(1, t)=u_{x x}(0, t)=u_{x x}(1, t)=0,
$$

the Dirichlet boundary conditions for $v$ and $\theta$

$$
v(0, t)=v(1, t)=\theta(0, t)=\theta(1, t)=0,
$$

and the Neumann one for $\varphi$

$$
\varphi_{x}(0, t)=\varphi_{x}(1, t)=0 .
$$

This latter condition and the absence of a source term in the last equation of (1.1) follow from the assumption that the cable is thermally insulated.

Remark 1.1

It is worth noting that different boundary conditions are physically significant as well, for instance, clamped rather than hinged ends or mixed conditions (one end clamped and the other one hinged) for the variable $u$, and Dirichlet boundary conditions for the variable $\varphi$. However, in this case, the analysis carried out in the present work requires major modifications, and the proofs become much more technical. In this situation, existence (and regularity) of attractors for the associated solution semigroup is an open problem at the moment and might be the object of future investigations.

System (1.1) models a one-dimensional suspension bridge with thermal effects and prescribed axial load $\beta$. The suspension cable (string) and the roadbed (beam) are thermoelastic and are coupled by means of a distributed system of one-sided springs. In particular, the roadbed equation contains a geometric nonlinearity accounting for elastic extensions, so that the resulting system is doubly nonlinear. When the suspension cable and the suspenders are neglected, the model reduces to a nonlinear parabolic system describing thermoelastic extensible beams, whose solution semigroup is known to possess a rich set of steady states [1] and a regular global attractor [2].

Actually, a more general and realistic approach can be performed by including also the width of the roadbed and the torsional oscillations. In this connection, a two-dimensional suspension bridge should be modeled as a long and narrow vibrating plate, coupled with a pair of main cables by means of two series of nonlinear suspenders fixed at the lateral sides (e.g., [3,4]). The resulting system turns out to be too hard to handle if nonlinearities occur; this is the reason why we only deal with the one-dimensional situation. However, although simpler, our model (1.1) is somehow challenging from a mathematical viewpoint, because no mechanical dissipation is present in the equations. In particular, the asymptotic analysis is highly nontrivial and requires the exploitation of certain dissipation integrals and sharp energy-type estimates. In addition, unlike some previous papers on cable-beam systems involving the coupling between parabolic and hyperbolic equations $[5,6]$, we are able here to obtain existence and optimal regularity of the global attractor without resorting to a bootstrap argument.

\subsection{Plan of the paper}

In Section 2, we discuss some earlier contributions on the long-term properties of string-beam systems modeling suspension bridges. In particular, we stress that all the previous results take advantage of the dissipation due to some external mechanical damping. In Section 3, we introduce the functional setting, while Section 4 is devoted to the well-posedness of the problem. In Section 5 , we dwell on the dissipative character of the system, witnessed by the existence of bounded absorbing sets. Finally, Section 6 deals with the main result about existence and regularity of global attractors.

\section{Earlier results on string-beam models of suspension bridges}

In recent years, an increasing attention has been payed to the analysis of buckling, vibrations, and post-buckling dynamics of nonlinear suspension bridge models (e.g., [7-10]). To the best of our knowledge, the major part of the papers published in the field deals with approximations and numerical simulations. Only few works are devoted to derive exact solutions, at least under stationary conditions $[11,12]$, and to scrutinize their periodic or global dynamics by analytical methods (see, e.g., [3] and references therein). This section is devoted to a brief survey on the subject. We begin by sketching out and discussing some linear and nonlinear models (with mechanical damping) that appeared so far in the literature.

\subsection{String-beam models for suspension bridges}

In the nineties, Glover, Lazer, McKenna, and Walter proposed and studied a nowadays well-known suspension bridge model where the coupling of the span with the main cable is taken into account [13-15]. Specifically, they considered the following damped system:

$$
\left\{\begin{array}{l}
\rho_{1} u_{t t}+\delta_{1} u_{x x x x}+v_{1} u_{t}+k(u-v)^{+}=f, \\
\rho_{2} v_{t t}-\delta_{2} v_{x x}+v_{2} v_{t}-k(u-v)^{+}=g .
\end{array}\right.
$$

The first equation of (2.1) describes the vertical oscillations of a one-dimensional beam, which represents the center span of the road bed, hanging by elastic cable stays. The second equation models the vertical vibrations of the main cable, whose ends are fixed to the pair of lateral piers. Accordingly, $u$ and $v$ represent the downward deflection in the vertical plane of the deck midline and the suspension main cable, respectively, with respect to their reference configurations. The constants $\rho_{1}, \rho_{2}>0$ are the mass densities per 
unit length of the roadbed and the cable, while $\delta_{1}, \delta_{2}>0$ are the flexural rigidity of the beam and the coefficient of tensile strength of the suspension string. Moreover, the terms $v_{1} u_{t}$ and $v_{2} v_{t}$, with $v_{1}, v_{2}>0$, account for an external resistance force linearly depending on the velocity and acting on the string-beam system. The sources $f$ and $g$ are (given) vertical dead-load distributions acting on the deck and the main cable, respectively. The nonlinear terms $\pm k(u-v)^{+}$model the restoring force due to the suspenders, which are assumed to behave as one-sided springs, with $k>0$ being the common stiffness. Such a restoring force is proportional to the elongation of the suspenders if they are stretched and vanishes if they are compressed. In addition, it holds the roadbed up and pulls the cable down; therefore, into the first equation, the plus sign in front of $k(u-v)^{+}$occurs, but into the second equation, the sign in front of the same term is minus. This model is quite naive: it neglects torsional effects and the influence of the side-part and pier deformations. Nevertheless, statics and dynamics of $(2.1)$ are nontrivial (e.g., $[7,11])$. The existence of a global attractor for the semigroup generated by this system has been recently obtained in $[16,17]$.

\subsection{String-beam models with large deflections}

Actually, if large deflections occur, the Lazer-McKenna model becomes inadequate, and the extensibility of the deck has to be considered. This can be performed by introducing a geometric nonlinear term, as proposed in the fifties by Woinowsky-Krieger [18], in order to account for a nonlinear dependence of the axial strain on the deformation gradient. This is of some importance in the modeling of large deflections of both suspension and cable-stayed bridges (e.g., [10]). Considering jointly the suspended bridge and the cable, we end up with the following system:

$$
\left\{\begin{array}{l}
\rho_{1} u_{t t}+\delta_{1} u_{x x x x}+v_{1} u_{t}-\left(\beta+\left\|u_{x}\right\|_{L^{2}(0,1)}^{2}\right) u_{x x}+k(u-v)^{+}=f \\
\rho_{2} v_{t t}-\delta_{2} v_{x x}+v_{2} v_{t}-k(u-v)^{+}=g
\end{array}\right.
$$

As previously stated, $v$ measures the displacement of the main cable, and $u$ represents the bending displacement of the roadbed of the bridge. The existence of nontrivial steady states and regular global attractors has been proved in [19], [5], and [20].

Other realistic models can be constructed by considering a more general form $F$ of the restraining force experienced by both the roadbed and the suspension cable as transmitted through the tie lines (stays). If this is the case, the previous system takes the form

$$
\left\{\begin{array}{l}
\rho_{1} u_{t t}+\delta_{1} u_{x x x x}+v_{1} u_{t}-\left(\beta+\left\|u_{x}\right\|_{L^{2}(0,1)}^{2}\right) u_{x x}+F\left(u-v, u_{t}-v_{t}\right)=f \\
\rho_{2} v_{t t}-\delta_{2} v_{x x}+v_{2} v_{t}-F\left(u-v, u_{t}-v_{t}\right)=g
\end{array}\right.
$$

When $F\left(\xi_{1}, \xi_{2}\right)=k \xi_{1}^{+}$, we recover at once (2.2). Assuming $F$ to represent the action of one-sided elastic springs and viscous dampers, the corresponding solution semigroup is known to possess a regular global attractor [6]. On the other hand, there are some cases in which $F$ can be reasonably assumed to be linear (e.g., [21]). Indeed, let the roadbed be supported by a symmetrical system of one-sided elastic springs (cable stays), each of which fastened on two symmetrically placed main (suspension) elastic cables, one above and one below the roadbed. Then, the (damped) dynamics of the resulting suspension bridge is described by the following system:

$$
\left\{\begin{array}{l}
\rho_{1} u_{t t}+\delta_{1} u_{x x x x}+v_{1} u_{t}-\left(\beta+\left\|u_{x}\right\|_{L^{2}(0,1)}^{2}\right) u_{x x}+k(u-v)+h\left(u_{t}-v_{t}\right)=f, \\
\rho_{2} v_{t t}-\delta_{2} v_{x x}+v_{2} v_{t}-k(u-v)-h\left(u_{t}-v_{t}\right)=g,
\end{array}\right.
$$

with $h \geq 0$. If the set of cables stays below the road bed is removed and $h=0$, then (2.4) turns into the previous model (2.2).

\section{Remark 2.1}

We stress that the main novelty of the system considered in this paper with respect to the existing literature on string-beam models of suspension bridges lies in the fact that the dissipation is entirely provided by the two heat equations. Hence, it seems interesting to investigate and extend the analysis to other types of temperature equations (e.g., Maxwell-Cattaneo or Gurtin-Pipkin [22, 23]) and/or to different mechanical nonlinearities. This will be the goal of a future work.

\section{Preliminaries}

\subsection{Functional setting and notation}

In what follows, $\langle\cdot, \cdot\rangle$ and $\|\cdot\|$ will denote the standard inner product and norm on the Hilbert space $\mathrm{H}=L^{2}(0,1)$. Introducing the biharmonic operator $A: \mathfrak{D}(A) \Subset \mathrm{H} \rightarrow \mathrm{H}$ defined as

$$
A u=u_{x x x x} \quad \text { with } \quad \mathfrak{D}(A)=\left\{u \in H^{4}(0,1): u(0)=u(1)=u_{x x}(0)=u_{x x}(1)=0\right\},
$$

we consider the compactly nested family of Hilbert spaces generated by the powers of $A$ ( $r$ will be always omitted whenever zero)

$$
\mathrm{H}_{r}=\mathfrak{D}\left(A^{\frac{r}{4}}\right), \quad\langle u, v\rangle_{r}=\left\langle A^{\frac{r}{4}} u, A^{\frac{r}{4}} v\right\rangle, \quad\|u\|_{r}=\left\|A^{\frac{r}{4}} u\right\|
$$

In particular,

$$
\mathrm{H}_{2}=H^{2}(0,1) \cap H_{0}^{1}(0,1) \Subset \mathrm{H}_{1}=H_{0}^{1}(0,1) \Subset \mathrm{H}=L^{2}(0,1) .
$$


Moreover, for $r>0$, it is understood that $\mathrm{H}_{-r}$ denotes the completion of the domain, so that $\mathrm{H}_{-r}$ is the dual space of $\mathrm{H}_{r}$. Accordingly, the symbol $\langle\cdot, \cdot\rangle$ also stands for duality product between $\mathrm{H}_{r}$ and $\mathrm{H}_{-r}$, and we have the generalized Poincaré inequalities

$$
\lambda_{1}\|u\|_{r}^{4} \leq\|u\|_{r+1}^{4}, \quad \forall u \in \mathrm{H}_{r+1}
$$

where $\lambda_{1}>0$ is the first eigenvalue of $A$. These inequalities, as well as the Hölder and the Young inequalities, will be tacitly used several times in the sequel. Finally, we consider the Hilbert space

$$
\hat{\mathrm{H}}=\left\{\varphi \in \mathrm{H}: \int_{0}^{1} \varphi(x) \mathrm{d} x=0\right\}
$$

of zero-mean functions, along with the space

$$
\hat{\mathrm{H}}_{1}=H^{1}(0,1) \cap \hat{\mathrm{H}}
$$

endowed with the gradient norm (because of the Poincaré inequality). The phase space of our problem will be

$$
\mathcal{H}=\mathrm{H}_{2} \times \mathrm{H} \times \mathrm{H}_{1} \times \mathrm{H} \times \mathrm{H} \times \hat{\mathrm{H}}
$$

normed by

$$
\|(u, \tilde{u}, v, \tilde{v}, \theta, \varphi)\|_{\mathcal{H}}^{2}=\|u\|_{2}^{2}+\|\tilde{u}\|^{2}+\|v\|_{1}^{2}+\|\tilde{v}\|^{2}+\|\theta\|^{2}+\|\varphi\|^{2} .
$$

We will also encounter the more regular space

$$
\mathcal{V}=\mathrm{H}_{4} \times \mathrm{H}_{2} \times \mathrm{H}_{2} \times \mathrm{H}_{1} \times \mathrm{H}_{2} \times \hat{\mathrm{H}}_{1}
$$

Remark 3.1

Along the paper, we will perform several formal energy-type estimates, which can be rigorously justified in a Galerkin approximation scheme.

\subsection{Two technical lemmas}

The first is a slightly modified version of the standard Gronwall lemma.

Lemma 3.2

Let $\Lambda:[0, \infty) \rightarrow[0, \infty)$ be an absolutely continuous function satisfying for some $\varkappa>0$ and almost every $t$ the inequality

$$
\frac{\mathrm{d}}{\mathrm{d} t} \Lambda(t)+2 \varkappa \Lambda(t) \leq \psi(t) \Lambda(t)
$$

where $\psi:[0, \infty) \rightarrow[0, \infty)$ fulfills

$$
\int_{\tau}^{t} \psi(y) d y \leq \varkappa(t-\tau)+m
$$

for every $t>\tau \geq 0$ and some $m \geq 0$. Then

$$
\Lambda(t) \leq \Lambda(0) \mathrm{e}^{m} \mathrm{e}^{-\varkappa t}
$$

Next, we report the following Gronwall-type lemma with parameters from the recent paper [24].

Lemma 3.3

Given $\varepsilon_{0}>0$, let $\Lambda_{\varepsilon}:[0, \infty) \rightarrow[0, \infty)$ be a family of absolutely continuous functions satisfying for every $\varepsilon \in\left(0, \varepsilon_{0}\right]$ the inequalities

$$
\frac{1}{2} E \leq \Lambda_{\varepsilon} \leq 2 E+k \frac{\mathrm{d}}{\mathrm{d} t} \Lambda_{\varepsilon}+\varepsilon \Lambda_{\varepsilon} \leq C \varepsilon^{2} \Lambda_{\varepsilon}^{3 / 2}+C
$$

for some $k \geq 0, C \geq 0$ and $E:[0, \infty) \rightarrow[0, \infty)$ continuous. Then there are constants $\delta>0, R \geq 0$ and an increasing function $\mathfrak{I} \geq 0$ such that

$$
E(t) \leq \Im(E(0)) \mathrm{e}^{-\delta t}+R
$$

\section{The dynamical system}

We begin with a well-posedness result.

Theorem 4.1

For all initial data $z \in \mathcal{H}$, problem (1.1) admits a unique weak ${ }^{\ddagger}$ solution

${ }^{\ddagger}$ Namely, all the equations of system (1.1) are satisfied in the (usual) weak sense. 


$$
Z \in \mathcal{C}([0, \infty), \mathcal{H})
$$

Moreover, given any pair of initial data $z_{1}, z_{2} \in \mathcal{H}$ such that $\left\|z_{i}\right\|_{\mathcal{H}} \leq R$, the corresponding solutions fulfill the continuous dependence estimate

$$
\left\|Z_{1}(t)-Z_{2}(t)\right\|_{\mathcal{H}} \leq \mathrm{e}^{\mathcal{Q}(R) t}\left\|z_{1}-z_{2}\right\|_{\mathcal{H}}, \quad \forall t \geq 0
$$

for some positive increasing function $\mathcal{Q}$.

The proof of this result is based on a standard Galerkin approximation procedure together with the use of a slight generalized version of the Gronwall lemma (cf. [25]) and is not reported here. As a consequence, system (1.1) generates a strongly continuous semigroup

$$
S(t): \mathcal{H} \rightarrow \mathcal{H}
$$

acting as

$$
z=\left(u_{0}, u_{1}, v_{0}, v_{1}, \theta_{0}, \varphi_{0}\right) \mapsto S(t) z=Z(t),
$$

where $Z(t)$ is the unique solution to (1.1) with initial datum $Z(0)=z$.

Next, we define the energy at time $t \geq 0$ corresponding to the initial datum $z \in \mathcal{H}$ as

$$
E(t)=\frac{1}{2}\|S(t) z\|_{\mathcal{H}}^{2}+\frac{1}{4}\left(\beta+\|u(t)\|_{1}^{2}\right)^{2}+\frac{1}{2}\left\|(u(t)-v(t))^{+}\right\|^{2},
$$

and we multiply the first equation of (1.1) by $u_{t}$, the second by $v_{t}$, the third by $\theta$, and the fourth by $\varphi$. Summing up, we find the energy identity (valid for all sufficiently regular initial data)

$$
\frac{\mathrm{d}}{\mathrm{d} t} E+\|\theta\|_{1}^{2}+\|\varphi\|_{1}^{2}=\left\langle f, u_{t}\right\rangle+\left\langle g, v_{t}\right\rangle+\langle h, \theta\rangle,
$$

where we made use of the relations

$$
\frac{1}{4} \frac{\mathrm{d}}{\mathrm{d} t}\left(\beta+\|u(t)\|_{1}^{2}\right)^{2}=-\left(\beta+\|u(t)\|_{1}^{2}\right)\left\langle u_{x x}(t), u_{t}(t)\right\rangle
$$

and

$$
\frac{1}{2} \frac{\mathrm{d}}{\mathrm{d} t}\left\|(u(t)-v(t))^{+}\right\|^{2}=\left\langle(u(t)-v(t))^{+}, u_{t}(t)-v_{t}(t)\right\rangle
$$

(e.g., $[2,5])$.

Remark 4.2

Neglecting the last two equations of (1.1) and performing similar calculations, one can easily see that the mechanical part of the system is conservative.

\section{Absorbing sets}

In this section, we prove the existence of an absorbing set for the semigroup $S(t)$. By definition, this is a bounded set $\mathbb{B} \subset \mathcal{H}$ with the following property: for any bounded set $\mathcal{B} \subset \mathcal{H}$, there exists an entering time $t_{\mathrm{e}}=t_{\mathrm{e}}(\mathcal{B}) \geq 0$ such that

$$
S(t) \mathcal{B} \subset \mathbb{B}, \quad \forall t \geq t_{\mathrm{e}}
$$

The absorbing set $\mathbb{B}$ is called invariant under the action of the semigroup if

$$
S(t) \mathbb{B} \subset \mathbb{B}, \quad \forall t \geq 0 .
$$

Remark 5.1

Once we have $\mathbb{B}$, we can always construct the invariant absorbing set

$$
\bigcup_{t \geq t_{\mathrm{e}}} s(t) \mathbb{B} \subset \mathbb{B}, \quad t_{\mathrm{e}}=t_{\mathrm{e}}(\mathbb{B})
$$

The existence of an invariant absorbing set for $S(t)$ is an immediate consequence of the next result. 
Theorem 5.2

We have the dissipative estimate

$$
E(t) \leq \Im(E(0)) \mathrm{e}^{-\delta t}+R
$$

for some structural quantities $\delta>0, R \geq 0$ and $\mathfrak{I}:[0, \infty) \rightarrow[0, \infty)$ increasing.

Proof

Along the proof, $C \geq 0$ will denote a generic constant depending on the structural quantities of the problem but independent of the initial energy $E(0)$. In light of (4.1), the functional

$$
\mathcal{L}(t)=E(t)-\langle f, u(t)\rangle-\langle g, v(t)\rangle
$$

fulfills the differential equality

Observing that

$$
\frac{\mathrm{d}}{\mathrm{d} t} \mathcal{L}+\|\theta\|_{1}^{2}+\|\varphi\|_{1}^{2}=\langle h, \theta\rangle .
$$

we obtain

$$
\langle h, \theta\rangle \leq \frac{1}{2}\|\theta\|_{1}^{2}+C,
$$

$$
\frac{\mathrm{d}}{\mathrm{d} t} \mathcal{L}+\frac{1}{2}\|\theta\|_{1}^{2}+\|\varphi\|_{1}^{2} \leq C .
$$

Next, defining the primitive ${ }^{\S}$

we introduce the auxiliary functional

$$
\hat{\varphi}(x, t)=\int_{0}^{x} \varphi(y, t) d y,
$$

$$
\Sigma(t)=\left\langle v_{t}(t), \hat{\varphi}(t)\right\rangle .
$$

Here and in all the proofs carried out in the paper, we deal with proper energy-type functionals satisfying differential equalities that follow in a straightforward way from system (1.1). For instance, computing the time derivative of $\Sigma$,

$$
\frac{\mathrm{d}}{\mathrm{d} t} \Sigma=\left\langle v_{t t}, \hat{\varphi}\right\rangle+\left\langle v_{t}, \hat{\varphi}_{t}\right\rangle=\left\langle v_{x x}+(u-v)^{+}-\varphi_{x}+g, \hat{\varphi}\right\rangle+\left\langle v_{t}, \varphi_{x}-v_{t}\right\rangle
$$

and thus

$$
\frac{\mathrm{d}}{\mathrm{d} t} \Sigma+\left\|v_{t}\right\|^{2}=-\left\langle v_{x}, \varphi\right\rangle+\left\langle(u-v)^{+}, \hat{\varphi}\right\rangle+\|\varphi\|^{2}+\langle g, \hat{\varphi}\rangle+\left\langle v_{t}, \varphi_{x}\right\rangle
$$

For clarity of presentation, these details will be often omitted, using expressions like by means of direct computations or by direct calculations. Because the right-hand side of the previous identity is controlled by

$$
\frac{1}{8}\left\|v_{t}\right\|^{2}+\frac{1}{16}\|v\|_{1}^{2}+\frac{1}{16}\left\|(u-v)^{+}\right\|^{2}+C\|\varphi\|_{1}^{2}+C
$$

we arrive at the differential inequality

$$
\frac{\mathrm{d}}{\mathrm{d} t} \Sigma+\frac{7}{8}\left\|v_{t}\right\|^{2} \leq \frac{1}{16}\|v\|_{1}^{2}+\frac{1}{16}\left\|(u-v)^{+}\right\|^{2}+C\|\varphi\|_{1}^{2}+C .
$$

At this point, we consider the further functionals

$$
\begin{aligned}
& \Upsilon(t)=\left\langle u_{t}(t), u(t)\right\rangle+\left\langle v_{t}(t), v(t)\right\rangle, \\
& \Psi(t)=\left\langle u_{t}(t), \theta(t)\right\rangle_{-1} .
\end{aligned}
$$

Concerning $\Upsilon$, we have

Estimating

$$
\begin{gathered}
\frac{\mathrm{d}}{\mathrm{d} t} \Upsilon+\|u\|_{2}^{2}+\|v\|_{1}^{2}+\left(\beta+\|u\|_{1}^{2}\right)^{2}-\beta\left(\beta+\|u\|_{1}^{2}\right)+\left\|(u-v)^{+}\right\|^{2} \\
=\left\|u_{t}\right\|^{2}+\left\|v_{t}\right\|^{2}-\left\langle u_{x x}, \theta\right\rangle-\left\langle v, \varphi_{x}\right\rangle+\langle f, u\rangle+\langle g, v\rangle .
\end{gathered}
$$

and

we infer that

$$
-\left\langle u_{x x}, \theta\right\rangle-\left\langle v, \varphi_{x}\right\rangle+\langle f, u\rangle+\langle g, v\rangle \leq \frac{1}{4}\|u\|_{2}^{2}+\frac{1}{4}\|v\|_{1}^{2}+C\|\theta\|_{1}^{2}+C\|\varphi\|_{1}^{2}+C
$$

$$
\frac{1}{2}\left(\beta+\|u\|_{1}^{2}\right)^{2}-\beta\left(\beta+\|u\|_{1}^{2}\right)=\frac{1}{2}\|u\|_{1}^{4}-\frac{1}{2} \beta^{2} \geq-C
$$

$$
\begin{aligned}
\frac{\mathrm{d}}{\mathrm{d} t} \Upsilon+ & \frac{3}{4}\|u\|_{2}^{2}+\frac{3}{4}\|v\|_{1}^{2}+\frac{1}{2}\left(\beta+\|u\|_{1}^{2}\right)^{2}+\left\|(u-v)^{+}\right\|^{2} \\
& \leq\left\|u_{t}\right\|^{2}+\left\|v_{t}\right\|^{2}+C\|\theta\|_{1}^{2}+C\|\varphi\|_{1}^{2}+C
\end{aligned}
$$

$\S$ In particular, $\hat{\varphi} \in H_{0}^{1}(0,1)$. 
Regarding $\Psi$,

We easily see that

$$
\begin{aligned}
\frac{\mathrm{d}}{\mathrm{d} t} \Psi+\left\|u_{t}\right\|^{2}= & \|\theta\|^{2}-\left\langle u_{t}, \theta\right\rangle-\left\langle(u-v)^{+}, \theta\right\rangle_{-1}+\left\langle u_{t}, h\right\rangle_{-1} \\
& +\langle f, \theta\rangle_{-1}+\left\langle u_{x x}, \theta\right\rangle-\left(\beta+\|u\|_{1}^{2}\right)\langle u, \theta\rangle .
\end{aligned}
$$

Moreover, because

$$
\begin{gathered}
\|\theta\|^{2}-\left\langle u_{t}, \theta\right\rangle-\left\langle(u-v)^{+}, \theta\right\rangle_{-1}+\left\langle u_{t}, h\right\rangle_{-1}+\langle f, \theta\rangle_{-1}+\left\langle u_{x x}, \theta\right\rangle \\
\leq \frac{1}{8}\left\|u_{t}\right\|^{2}+\frac{1}{16}\|u\|_{2}^{2}+\frac{1}{16}\left\|(u-v)^{+}\right\|^{2}+C\|\theta\|_{1}^{2}+C .
\end{gathered}
$$

we obtain

$$
\|u\|_{1} \leq C\left|\beta+\|u\|_{1}^{2}\right|^{1 / 2}+C|\beta|^{1 / 2} \leq C E^{1 / 4}+C
$$

Thus, we conclude that

$$
\begin{aligned}
-\left(\beta+\|u\|_{1}^{2}\right)\langle u, \theta\rangle & \leq C\|\theta\|\left(\|u\|_{1}^{3}+1\right) \\
& \leq C\|\theta\| E^{3 / 4}+C\|\theta\| \\
& \leq C\|\theta\| E^{3 / 4}+C\|\theta\|_{1}^{2}+C .
\end{aligned}
$$

$$
\frac{\mathrm{d}}{\mathrm{d} t} \Psi+\frac{7}{8}\left\|u_{t}\right\|^{2} \leq \frac{1}{16}\|u\|_{2}^{2}+\frac{1}{16}\left\|(u-v)^{+}\right\|^{2}+C\|\theta\| E^{3 / 4}+C\|\theta\|_{1}^{2}+C .
$$

Finally, for $\varepsilon>0$ and $k \geq 0$, we write

$$
\Lambda_{\varepsilon}(t)=\mathcal{L}(t)+4 \varepsilon \Upsilon(t)+8 \varepsilon\{\Psi(t)+\Sigma(t)\}+k,
$$

where $k$ is large enough and $\varepsilon$ is small enough such that

$$
\frac{1}{2} E(t) \leq \Lambda_{\varepsilon}(t) \leq 2 E(t)+k
$$

Then, in light of inequalities (5.1)-(5.4) and (5.5), there exists $0<\varepsilon_{0}<1$ such that, for every $\varepsilon \in\left(0, \varepsilon_{0}\right]$,

$$
\begin{aligned}
\frac{\mathrm{d}}{\mathrm{d} t} \Lambda_{\varepsilon}+\varepsilon \Lambda_{\varepsilon}+\frac{1}{4}\left(1-C_{\varepsilon}\right)\left[\|\theta\|_{1}^{2}+\|\varphi\|_{1}^{2}\right] & \leq C \varepsilon\|\theta\| E^{3 / 4}+C \\
& \leq C \varepsilon^{2} \Lambda_{\varepsilon}^{3 / 2}+\frac{1}{8}\|\theta\|_{1}^{2}+C .
\end{aligned}
$$

Therefore, possibly reducing $\varepsilon_{0}>0$, we end up with

$$
\frac{\mathrm{d}}{\mathrm{d} t} \Lambda_{\varepsilon}+\varepsilon \Lambda_{\varepsilon} \leq C \varepsilon^{2} \Lambda_{\varepsilon}^{3 / 2}+C
$$

Exploiting once more (5.5), an application of Lemma 3.3 completes the proof.

\section{Remark 5.3}

It is worth noting that, up to minor modifications, the conclusion of Theorem 5.2 holds true also, allowing the presence of timedependent source terms $f$ and $h$ in system (1.1). Indeed, the additional terms that pop up in the estimates can be actually handled as in [2].

\section{The global attractor}

\subsection{Statement of the result}

By definition, the global attractor of $S(t)$ is the unique compact set $\mathbb{A} \subset \mathcal{H}$, which is at the same time

- fully invariant: $S(t) \mathbb{A}=\mathbb{A}$ for every $t \geq 0$ and

- attracting : $\lim _{t \rightarrow \infty} \boldsymbol{\delta}_{\mathcal{H}}(S(t) \mathcal{B}, \mathbb{A})=0$ for any bounded set $\mathcal{B} \subset \mathcal{H}$.

In the usual notation, $\boldsymbol{\delta}_{\mathcal{H}}\left(\mathcal{B}_{1}, \mathcal{B}_{2}\right)$ denotes the standard Hausdorff semidistance between two nonempty sets $\mathcal{B}_{1}, \mathcal{B}_{2} \subset \mathcal{H}$.

\section{Theorem 6.1}

The semigroup $S(t): \mathcal{H} \rightarrow \mathcal{H}$ possesses the connected global attractor $\mathbb{A}$ contained and bounded in $\mathcal{V}$.

Remark 6.2

In a Banach space setting, the global attractor of a strongly continuous semigroup $S(t)$ is always a connected set. We refer the reader to the classical books [26-29] for more details.

Remark 6.3

Within our hypotheses on the forcing terms, the regularity of $\mathbb{A}$ is optimal. However, it is possible to prove that the attractor $\mathbb{A}$ is as regular as $f, g, h$ permit. 
The remaining part of the paper is devoted to the proof of Theorem 6.1. In what follows, $\mathbb{B}$ will denote a fixed invariant absorbing set for $S(t)$, and $C=C(\mathbb{B}) \geq 0$ will stand for a generic constant depending only on $\mathbb{B}$. In particular, we have the uniform bound

$$
\sup _{t \geq 0} \sup _{z \in \mathbb{B}}\|S(t) z\|_{\mathcal{H}} \leq C
$$

Up to a translation, we can also assume $h \equiv 0$ (e.g., [2]).

\subsection{Dissipation integrals}

We need a dissipation integral for the norm of $u_{t}$.

Theorem 6.2

For every $v>0$ small, the integral estimate

$$
\int_{\tau}^{t}\left\|u_{t}(y)\right\|^{2} \mathrm{~d} y \leq v(t-\tau)+\frac{C}{v}
$$

holds for all $t>\tau \geq 0$ and all initial data in $\mathbb{B}$.

Proof

The functional $\mathcal{L}$ introduced in the proof of Theorem 5.2 satisfies the identity (recall that $h \equiv 0$ )

$$
\frac{\mathrm{d}}{\mathrm{d} t} \mathcal{L}+\|\theta\|_{1}^{2}+\|\varphi\|_{1}^{2}=0
$$

Therefore, integrating on $[0, t]$ and exploiting (6.1), we infer that

$$
\int_{0}^{\infty}\|\theta(y)\|_{1}^{2} \mathrm{~d} y \leq C .
$$

Referring once more to the proof of Theorem 5.2, we consider the functional $\Psi$, which fulfills

$$
\begin{aligned}
\frac{\mathrm{d}}{\mathrm{d} t} \Psi+\left\|u_{t}\right\|^{2}= & \|\theta\|^{2}-\left\langle u_{t}, \theta\right\rangle-\left\langle(u-v)^{+}, \theta\right\rangle_{-1} \\
& +\langle f, \theta\rangle_{-1}+\left\langle u_{x x}, \theta\right\rangle-\left(\beta+\|u\|_{1}^{2}\right)\langle u, \theta\rangle .
\end{aligned}
$$

By means of (6.1), we can estimate

$$
-\left\langle u_{t}, \theta\right\rangle+\|\theta\|^{2}-\left\langle(u-v)^{+}, \theta\right\rangle_{-1}+\langle f, \theta\rangle_{-1}+\left\langle u_{x x}, \theta\right\rangle-\left(\beta+\|u\|_{1}^{2}\right)\langle u, \theta\rangle \leq v+\frac{C}{v}\|\theta\|_{1}^{2}
$$

for all positive $v \leq 1$, and hence

$$
\frac{\mathrm{d}}{\mathrm{d} t} \Psi+\left\|u_{t}\right\|^{2} \leq v+\frac{C}{v}\|\theta\|_{1}^{2}
$$

Integrating the last inequality on $[\tau, t]$ and using (6.1)-(6.2), we are finished.

\subsection{The decomposition}

In the same spirit of [30], we split the solution $S(t) z$ originated from initial data $z \in \mathbb{B}$ into the sum

$$
S(t) z=L(t) z+K(t) z
$$

where

$$
\begin{aligned}
& L(t) z=\left(w(t), w_{t}(t), p(t), p_{t}(t), \xi(t), \mu(t)\right) \\
& K(t) z=\left(r(t), r_{t}(t), q(t), q_{t}(t), \zeta(t), \eta(t)\right)
\end{aligned}
$$

solven

$$
\left\{\begin{array}{l}
w_{t t}+w_{x x x x}-\left(\beta+\|u\|_{1}^{2}\right) w_{x x}+\ell w+\xi_{x x}=0 \\
p_{t t}-p_{x x}+\mu_{x}=0 \\
\xi_{t}-\xi_{x x}-w_{t x x}=0 \\
\mu_{t}-\mu_{x x}+p_{t x}=0 \\
\left(w(0), w_{t}(0), p(0), p_{t}(0), \xi(0), \mu(0)\right)=z
\end{array}\right.
$$


and

$$
\left\{\begin{array}{l}
r_{t t}+r_{x x x x}-\left(\beta+\|u\|_{1}^{2}\right) r_{x x}+(u-v)^{+}-\ell w+\zeta_{x x}=f \\
q_{t t}-q_{x x}-(u-v)^{+}+\eta_{x}=g \\
\zeta_{t}-\zeta_{x x}-r_{t x x}=0 \\
\eta_{t}-\eta_{x x}+q_{t x}=0 \\
\left(r(0), r_{t}(0), q(0), q_{t}(0), \zeta(0), \eta(0)\right)=0 .
\end{array}\right.
$$

Here, $\ell=\ell(\mathbb{B})>0$ is a positive constant large enough in order to have

$$
\frac{1}{2}\|w\|_{2}^{2}+\ell\|w\|^{2}+\left(\beta+\|u\|_{1}^{2}\right)\|w\|_{1}^{2} \geq \frac{1}{4}\|w\|_{2}^{2} .
$$

This is possible because of (6.1) and the interpolation inequality

$$
\|w\|_{1}^{2} \leq\|w\|\|w\|_{2} .
$$

Moreover, it is also clear that $\mu(t)$ and $\eta(t)$ are zero-mean functions for every $t \geq 0$.

Lemma 6.5

There exists $\varkappa=\varkappa(\mathbb{B})>0$ such that

$$
\|L(t) z\|_{\mathcal{H}} \leq C \mathrm{e}^{-\varkappa t} .
$$

Proof

We denote for simplicity

By direct calculations, the functional

$$
E_{0}(t)=\|L(t) z\|_{\mathcal{H}}^{2}
$$

$$
\mathcal{L}_{0}(t)=E_{0}(t)+\ell\|w(t)\|^{2}+\left(\beta+\|u(t)\|_{1}^{2}\right)\|w(t)\|_{1}^{2}
$$

fulfills the identity

and thus, exploiting (6.1),

$$
\frac{\mathrm{d}}{\mathrm{d} t} \mathcal{L}_{0}+2\|\xi\|_{1}^{2}+2\|\mu\|_{1}^{2}=-2\left\langle u_{x x}, u_{t}\right\rangle\|w\|_{1}^{2}
$$

Then, we consider the auxiliary functionals

$$
\frac{\mathrm{d}}{\mathrm{d} t} \mathcal{L}_{0}+2\|\xi\|_{1}^{2}+2\|\mu\|_{1}^{2} \leq C\left\|u_{t}\right\| E_{0}
$$

where

$$
\begin{aligned}
& \Sigma_{0}(t)=\left\langle p_{t}(t), \hat{\mu}(t)\right\rangle \\
& \Upsilon_{0}(t)=\left\langle w_{t}(t), w(t)\right\rangle+\left\langle p_{t}(t), p(t)\right\rangle, \\
& \Psi_{0}(t)=\left\langle w_{t}(t), \xi(t)\right\rangle_{-1},
\end{aligned}
$$

$$
\hat{\mu}(x, t)=\int_{0}^{x} \mu(y, t) d y
$$

is the primitive of the function $\mu^{\|}$. We begin by estimating $\Sigma_{0}$ as

$$
\frac{\mathrm{d}}{\mathrm{d} t} \Sigma_{0}+\left\|p_{t}\right\|^{2}=\|\mu\|^{2}+\left\langle p_{t}, \mu_{x}\right\rangle-\left\langle p_{x}, \mu\right\rangle \leq \frac{1}{8}\left\|p_{t}\right\|^{2}+\frac{1}{16}\|p\|_{1}^{2}+C\|\mu\|_{1}^{2} .
$$

Next, concerning $\Upsilon_{0}$, we have

Lastly, thanks to (6.1), the functional $\Psi_{0}$ satisfies

$$
\begin{aligned}
\frac{\mathrm{d}}{\mathrm{d} t} \Upsilon_{0} & +\|w\|_{2}^{2}+\|p\|_{1}^{2}+\ell\|w\|^{2}+\left(\beta+\|u\|_{1}^{2}\right)\|w\|_{1}^{2} \\
& =\left\|w_{t}\right\|^{2}+\left\|p_{t}\right\|^{2}+\left\langle w_{x}, \xi_{x}\right\rangle-\left\langle p, \mu_{x}\right\rangle \\
& \leq \frac{1}{4}\|w\|_{2}^{2}+\frac{1}{4}\|p\|_{1}^{2}+\left\|w_{t}\right\|^{2}+\left\|p_{t}\right\|^{2}+C\|\mu\|_{1}^{2}+C\|\xi\|_{1}^{2} .
\end{aligned}
$$

$$
\begin{aligned}
\frac{\mathrm{d}}{\mathrm{d} t} \Psi_{0}+\left\|w_{t}\right\|^{2} & =\|\xi\|^{2}-\left\langle w_{t}, \xi\right\rangle+\left\langle w_{x x}, \xi\right\rangle-\left(\beta+\|u\|_{1}^{2}\right)\langle w, \xi\rangle-\ell\langle w, \xi\rangle_{-1} \\
& \leq C\left\|w_{t}\right\|\|\xi\|_{1}+C\|w\|_{2}\|\xi\|_{1}+\|\xi\|^{2} \\
& \leq \frac{1}{8}\left\|w_{t}\right\|^{2}+\frac{1}{16}\|w\|_{2}^{2}+C\|\xi\|_{1}^{2} .
\end{aligned}
$$

At this point, for $\varepsilon \in(0,1]$, we set

$$
\Lambda_{0}(t)=\mathcal{L}_{0}(t)+4 \varepsilon \Upsilon_{0}(t)+8 \varepsilon\left\{\Psi_{0}(t)+\Sigma_{0}(t)\right\} .
$$




It is apparent from (6.1) and (6.5) that, for $\varepsilon>0$ sufficiently small,

$$
\frac{1}{2} E_{0}(t) \leq \Lambda_{0}(t) \leq C E_{0}(t)
$$

Moreover, collecting (6.6)-(6.9) and exploiting (6.5) once more, for every $\varepsilon>0$ small enough, the functional $\Lambda_{0}$ fulfills the differential inequality

Thus, in light of (6.10), we end up with

$$
\frac{\mathrm{d}}{\mathrm{d} t} \Lambda_{0}+\varepsilon E_{0} \leq C\left\|u_{t}\right\| E_{0} \leq \frac{\varepsilon}{2} E_{0}+\frac{C}{\varepsilon}\left\|u_{t}\right\|^{2} E_{0}
$$

$$
\frac{\mathrm{d}}{\mathrm{d} t} \Lambda_{0}+2 \varkappa \Lambda_{0} \leq C\left\|u_{t}\right\|^{2} \Lambda_{0}
$$

for some $\varkappa=\varkappa(\mathbb{B})>0$. Up to fixing $v>0$ in Theorem 6.2 sufficiently small in order to have

$$
\int_{\tau}^{t}\left\|u_{t}(y)\right\|^{2} \mathrm{~d} y \leq \varkappa(t-\tau)+C
$$

appealing to Lemma 3.2 and (6.10) the claim follows.

Lemma 6.6

We have the uniform estimate

$$
\sup _{t \geq 0}\|K(t) z\|_{\mathcal{V}} \leq C
$$

Proof

We begin by observing that, due to (6.1) and Lemma 6.5 , the following estimate holds

$$
\|r\|_{3}^{2} \leq\|r\|_{2}\|r\|_{4} \leq\left[\|w\|_{2}+\|u\|_{2}\right]\|r\|_{4} \leq C\|r\|_{4}
$$

Next, setting

we consider the functional

$$
E_{1}(t)=\|K(t) z\|_{\mathcal{V}}^{2}
$$

$$
\begin{aligned}
\mathcal{L}_{1}(t)= & E_{1}(t)+\left(\beta+\|u(t)\|_{1}^{2}\right)\|r(t)\|_{3}^{2}+2\left\langle(u(t)-v(t))^{+}, r_{x x x x}(t)\right\rangle \\
& +2\left\langle(u(t)-v(t))^{+}, q_{x x}(t)\right\rangle-2\left\langle f, r_{x x x x}(t)\right\rangle+2\left\langle g, q_{x x}(t)\right\rangle .
\end{aligned}
$$

By means of (6.1), (6.11), and Lemma 6.5, we have

$$
\begin{aligned}
\frac{\mathrm{d}}{\mathrm{d} t} \mathcal{L}_{1}+2\|\zeta\|_{3}^{2}+2\|\eta\|_{2}^{2}= & -2\left\langle u_{t}, u_{x x}\right\rangle\|r\|_{3}^{2}+2\left\langle\left[(u-v)^{+}\right]_{t}, r_{x x x x}\right\rangle \\
& +2\left\langle\left[(u-v)^{+}\right]_{t}, q_{x x}\right\rangle+2 \ell\left\langle w_{x x}, r_{t x x}\right\rangle \\
\leq & C\|r\|_{3}^{2}+C\left[\left\|u_{t}\right\|+\left\|v_{t}\right\|\right]\left[\|r\|_{4}+\|q\|_{2}\right]+C\left\|r_{t}\right\|_{2} \\
\leq & C\|r\|_{4}+C\|q\|_{2}+C\left\|r_{t}\right\|_{2} .
\end{aligned}
$$

At this point, we introduce the further functionals

$$
\begin{aligned}
& \Sigma_{1}(t)=-\left\langle q_{t}(t), \eta_{x}(t)\right\rangle, \\
& \Upsilon_{1}(t)=\left\langle r_{t}(t), r(t)\right\rangle_{2}+\left\langle q_{t}(t), q(t)\right\rangle_{1}, \\
& \Psi_{1}(t)=\left\langle r_{t}(t), \zeta(t)\right\rangle_{1}
\end{aligned}
$$

that will be estimated using (6.1), (6.11), and Lemma 6.5 without explicit mention. Regarding $\Sigma_{1}$, we have

$$
\begin{aligned}
\frac{\mathrm{d}}{\mathrm{d} t} \Sigma_{1}+\left\|q_{t}\right\|_{1}^{2} & =\|\eta\|_{1}^{2}+\left\langle q_{t x}, \eta_{x x}\right\rangle-\left\langle q_{x x}, \eta_{x}\right\rangle-\left\langle(u-v)^{+}, \eta_{x}\right\rangle-\left\langle g, \eta_{x}\right\rangle \\
& \leq \frac{1}{8}\left\|q_{t}\right\|_{1}^{2}+\frac{1}{16}\|q\|_{2}^{2}+C\|\eta\|_{2}^{2}+C .
\end{aligned}
$$

Concerning $\Upsilon_{1}$, we infer that

$$
\begin{aligned}
\frac{\mathrm{d}}{\mathrm{d} t} \Upsilon_{1}+\|r\|_{4}^{2}+\|q\|_{2}^{2}+\|u\|_{1}^{2}\|r\|_{3}^{2}= & \left\|r_{t}\right\|_{2}^{2}+\left\|q_{t}\right\|_{1}^{2}-\beta\|r\|_{3}^{2}-\left\langle(u-v)^{+}, q_{x x}\right\rangle \\
& -\left\langle(u-v)^{+}, r_{x x x x}\right\rangle+\ell\left\langle w, r_{x x x x}\right\rangle-\left\langle\zeta_{x x}, r_{x x x x}\right\rangle \\
& +\left\langle\eta_{x}, q_{x x}\right\rangle+\left\langle f, r_{x x x x}\right\rangle-\left\langle g, q_{x x}\right\rangle \\
\leq & \frac{1}{4}\|r\|_{4}^{2}+\frac{1}{4}\|q\|_{2}^{2}+\left\|r_{t}\right\|_{2}^{2}+\left\|q_{t}\right\|_{1}^{2}+C\|\eta\|_{2}^{2}+C\|\zeta\|_{2}^{2}+C .
\end{aligned}
$$


Finally, the functional $\Psi_{1}$ fulfills

$$
\begin{aligned}
\frac{\mathrm{d}}{\mathrm{d} t} \Psi_{1}+\left\|r_{t}\right\|_{2}^{2}= & \|\zeta\|_{2}^{2}+\left\langle r_{x x}, \zeta\right\rangle_{2}-\left(\beta+\|u\|_{1}^{2}\right)\langle r, \zeta\rangle_{2}+\left\langle(u-v)^{+}, \zeta_{x x}\right\rangle \\
& -\ell\left\langle w, \zeta_{x x}\right\rangle-\left\langle r_{t}, \zeta\right\rangle_{2}-\left\langle f, \zeta_{x x}\right\rangle \\
\leq & \frac{1}{8}\left\|r_{t}\right\|_{2}^{2}+\frac{1}{16}\|r\|_{4}^{2}+C\|\zeta\|_{2}^{2}+C .
\end{aligned}
$$

At this point, for $\varepsilon>0$, we consider the functional

$$
\Lambda_{1}(t)=\mathcal{L}_{1}(t)+4 \varepsilon \Upsilon_{1}(t)+8 \varepsilon\left\{\Psi_{1}(t)+\Sigma_{1}(t)\right\},
$$

which, collecting (6.12)-(6.15), satisfies the inequality

$$
\begin{aligned}
\frac{\mathrm{d}}{\mathrm{d} t} \Lambda_{1}+\varepsilon E_{1}+(1-C \varepsilon)\left[\|\zeta\|_{3}^{2}+\|\eta\|_{2}^{2}\right] & +\varepsilon\|r\|_{4}^{2}+\varepsilon\|q\|_{2}^{2}+\varepsilon\left\|r_{t}\right\|_{2}^{2} \\
& \leq C\|r\|_{4}+C\|q\|_{2}+C\left\|r_{t}\right\|_{2}+C \\
& \leq \frac{\varepsilon}{2}\|r\|_{4}^{2}+\frac{\varepsilon}{2}\|q\|_{2}^{2}+\frac{\varepsilon}{2}\left\|r_{t}\right\|_{2}^{2}+\frac{C}{\varepsilon},
\end{aligned}
$$

for every $\varepsilon$ small enough. Moreover, it is apparent from (6.1) and (6.11) that, for all $\varepsilon>0$ small,

$$
\frac{1}{2} E_{1}(t)-C \leq \Lambda_{1}(t) \leq C E_{1}(t)+C
$$

In conclusion, fixing $\varepsilon>0$ sufficiently small, we arrive at

$$
\frac{\mathrm{d}}{\mathrm{d} t} \Lambda_{1}+\kappa \Lambda_{1} \leq \mathrm{C}
$$

for some $\kappa>0$, and an application of the Gronwall lemma completes the proof.

\subsection{Conclusion of the proof of Theorem 6.1}

Collecting Lemmas 6.5 and 6.6 , there exists a closed ball $\mathcal{C} \subset \mathcal{V}$ such that

$$
\lim _{t \rightarrow \infty} \delta_{\mathcal{H}}(S(t) \mathbb{B}, \mathcal{C})=0 .
$$

Because the embedding $\mathcal{V} \Subset \mathcal{H}$ is compact, by classical arguments (e.g., [26, 29]), we have the existence of the global attractor $\mathbb{A} \subset \mathcal{C}$.

\section{References}

1. Bochicchio I, Giorgi C, Vuk E. Steady states analysis and exponential stability of an extensible thermoelastic system. Communication to SIMAI Congress 2009; 3:232-243.

2. Giorgi C, Naso MG, Pata V, Potomkin M. Global attractors for the extensible thermoelastic beam system. Journal of Differential Equations 2009; 246:3496-3517.

3. Drábek P, Holubová G, Matas A, Nečesal P. Nonlinear models of suspension bridges: discussion of the results. Applied Mathematics 2003; 48:497-514.

4. Holubová G, Matas A. Initial-boundary problem for the nonlinear string-beam system. Journal of Mathematical Analysis and Applications 2003; 288:784-802.

5. Bochicchio I, Giorgi C, Vuk E. Long-term dynamics of the coupled suspension bridge system. Mathematical Models \& Methods in Applied Sciences 2012; 22(1250021):22.

6. Bochicchio I, Giorgi C, Vuk E. Asymptotic dynamics of nonlinear coupled suspension bridge equations. Journal of Mathematical Analysis and Applications 2013; 402:319-333.

7. Choi QH, Jung T. A nonlinear suspension bridge equation with nonconstant load. Nonlinear Analysis 1999; 35:649-668.

8. Abdel-Ghaffar AM, Rubin LI. Non linear free vibrations of suspension bridges: theory. ASCE Journal Of Engineering Mechanics 1983; 109:313-329.

9. Abdel-Ghaffar AM, Rubin LI. Non linear free vibrations of suspension bridges: application. ASCE Journal Of Engineering Mechanics 1983; 109:330-345.

10. Kanok-Nukulchai W, Yiu PKA, Brotton DM. Mathematical modelling of cable-stayed bridges. Structural Engineering International 1992; 2:108-113.

11. An Y. Nonlinear perturbations of a coupled system of steady state suspension bridge equations. Nonlinear Analysis 2002; 51:1285-1292.

12. Coti Zelati M, Giorgi C, Pata V. Steady states of the hinged extensible beam with external load. Mathematical Models \& Methods in Applied Sciences 2010; 20:43-58.

13. Glover J, Lazer AC, McKenna PJ. Existence and stability of large scale nonlinear oscillations in suspension bridges. Zeitschrift fur Angewandte Mathematik und Physik 1989; 40:172-200.

14. Lazer AC, McKenna PJ. Large-amplitude periodic oscillations in suspension bridges: some new connections with nonlinear analysis. SIAM Review 1990; 32:537-578.

15. McKenna PJ, Walter W. Nonlinear oscillations in a suspension bridge. Archive for Rational Mechanics and Analysis 1987; 98:167-177.

16. Ma Q, Zhong C. Existence of strong solutions and global attractors for the coupled suspension bridge equations. Journal of Differential Equations 2009; 246:3755-3775.

17. Zhong C, Ma Q, Sun C. Existence of strong solutions and global attractors for the suspension bridge equations. Nonlinear Analysis 2007; 67:442-454. 
18. Woinowsky-Krieger S. The effect of an axial force on the vibration of hinged bars. Journal of Applied Mechanics 1950; 17:35-36.

19. Bochicchio I, Giorgi C, Vuk E. Long-term damped dynamics of the extensible suspension bridge. International Journal of Differential Equations 2010:Art. ID 383420, 19.

20. Giorgi C, Vuk E. Steady-state solutions for a suspension bridge with intermediate supports. Boundary Value Problems 2013; 2013:204.

21. Dell'Oro F, Giorgi C, Pata V. Asymptotic behavior of coupled linear systems modeling suspension bridges. Zeitschrift fur Angewandte Mathematik und Physik. (in press). doi: 10.1007/s00033-014-0414-9.

22. Cattaneo C. Sulla conduzione del calore. Atti del Seminario Matematico e Fisico dell'Università di Modena 1948; 3:83-101.

23. Gurtin ME, Pipkin AC. A general theory of heat conduction with finite wave speeds. Archive for Rational Mechanics and Analysis 1968; 31:113-126.

24. Pata V. Uniform estimates of Gronwall type. Journal of Mathematical Analysis and Applications 2011; 373:264-270.

25. Pata V, Prouse G, Vishik MI. Traveling waves of dissipative non-autonomous hyperbolic equations in a strip. Advances in Differential Equations 1998; 3:249-270

26. Babin AV, Vishik MI. Attractors of evolution equations. In Studies in Mathematics and its Applications, Vol. 25. Elsevier Science Publishers B.V.: Amsterdam, 1992.

27. Hale JK. Asymptotic Behavior of Dissipative Systems. American Mathematical Society: Providence, 1988.

28. Haraux A. Systèmes Dynamiques Dissipatifs et Applications. Masson: Paris, 1991.

29. Temam R. Infinite-dimensional Dynamical Systems in Mechanics and Physics. Springer: New York, 1997.

30. Giorgi C, Pata V, Vuk E. On the extensible viscoelastic beam. Nonlinearity 2008; 21:713-733. 